\title{
Factors Related to Early Recurrence of Idiopathic Clubfoot Post the Ponseti Method
}

\author{
Limpaphayom N, MD, Sailohit P*, MD \\ Department of Orthopaedics, Chulalongkorn University, Bangkok, Thailand \\ *Department of Orthopaedics, Police General Hospital, Bangkok, Thailand

This is an open-access article distributed under the terms of the Creative Commons Attribution License, which permits unrestricted use,
distribution, and reproduction in any medium, provided the original work is properly cited
Date of submission: 05th September 2018
Date of acceptance: 07 th May 2019

\begin{abstract}
Introduction: Idiopathic clubfoot or congenital talipes equinovarus (CTEV) is managed by the Ponseti method worldwide; however, the recurrence of the deformity is a challenging problem. The purpose was to review the factors associated with early recurrence of CTEV post the Ponseti method.

Materials and Methods: During 2011-2016, 34 infants with 52 CTEV, who underwent the Ponseti method and a minimum follow-up period of six months, were reviewed. Twenty-two infants (65\%) were male, and 18 infants (53\%) had bilateral CTEV. Recurrence of CTEV was defined as a reappearance of at least one of the four components of the deformity. The association between recurrence and factors, including age, gender, bilaterality, family geography, type of principal caregiver, severity at presentation, centre where the Ponseti method was initiated, compliance to foot abduction brace (FAB), practice of stretching exercise, type of FAB, and complications of casting, were evaluated using univariate logistic regression analysis.
\end{abstract}

Results: The median age at initiation of the treatment was 3.4 (IQR; 2.1-12.6) weeks. A median of six (range; 3-12) casts were required. Tenotomy was performed in 32/34 (94\%) of cases. Recurrence occurred in $14 / 52$ feet (27\%) at an average follow-up period of $2.3 \pm 1.1$ years. Noncompliance to $\mathrm{FAB}$ protocol began at an average age of $11.2 \pm 6.5$ months, and significantly increased the risk of recurrence during the weaning phase [OR $(95 \% \mathrm{CI})=8.4(1.2$ 92.4), $\mathrm{p}=0.03]$. Other factors were not associated with the recurrence.

Conclusion: Non-compliance to FAB occurred early during the treatment and related to a risk of recurrence of CTEV. Physicians should encourage the parents and/or guardians to follow the protocol to decrease the risk of recurrence.

\section{Key Words:}

braces, compliance, clubfoot, recurrence, treatment

\section{INTRODUCTION}

Idiopathic clubfoot or congenital talipes equinovarus (CTEV) is a well-known complex, congenital foot deformity composed of four components, namely: equinus, hindfoot varus, forefoot adduction and cavus ${ }^{1}$. Conservative treatment by manipulating and casting utilised the Ponseti method is the mainstay of practice and satisfactory results have been achieved by several authors ${ }^{2-6}$. The use of a foot abduction brace (FAB) after initial correction is mandatory to maintain the correction and to prevent the recurrence of $\mathrm{CTEV}^{1,6-9}$. However, this is a challenging process, which requires cooperation from the parents or guardians ${ }^{1,3,10,11}$.

The recurrence after initial correction of CTEV leads to further treatment and untoward outcomes. These include more casts, repeated tenotomy, and rarely more extensive surgical procedures which could affect the function of the patients in the long run'. Factors associated with the recurrence of CTEV could be the patient, physician, or caregiver-associated characteristics ${ }^{1,12-15}$. Non-modifiable factors include high grade of deformity, ethnicity, and parental educational level $1^{1,6,12,16}$. An important modifiable factor is non-compliance to FAB or practice of stretching exercise ${ }^{1,6,9,15,16}$. Physicians managing patients with CTEV should be aware of these factors before initiating the Ponseti method.

At our institution, the selective soft tissue release procedure ${ }^{17}$ become less favorable as we adopt the Ponseti method as a standard treatment for CTEV since 2011. The purpose of this study was to investigate clinical factors related to the recurrence of CTEV after the Ponseti method.

\section{MATERIALS AND METHODS}

We retrospectively reviewed 34 medical records 22 males (65\%), 12 females (35\%)] from 2011 to 2016 of children

Corresponding Author: Noppachart Limpaphayom, Department of Orthopaedics, Faculty of Medicine, Chulalongkorn University, Pathumwan, Bangkok 10330, Thailand

Email: Noppachart.L@chula.ac.th 
who were treated for idiopathic CTEV at our institution with a minimum follow-up period of six months after the completion of the casting process. Eighteen patients (53\%) had bilateral CTEV while 16 patients (37\%) had unilateral CTEV. Clubfeet with neuromuscular or syndromic in origin were excluded.

All patients underwent the Ponseti method, including a weekly foot manipulation and serial casting followed by percutaneous tendoachilles tenotomy (pTAL) if indicated. The procedure was performed by one physician (N.L.). To maintain the correction, FAB was used as previously described $^{1,4}$. Types of FAB utilised in the study were a fixed (Denis Browne bar) type and an articulated (Dobbs bar) type ${ }^{4,18}$. The selection of FAB type was at parental discretion. Nineteen fixed type bars and 15 articulated type bars were used. The standard FAB protocol was administered regardless of bar type. The FAB was worn full time (23 hours) in the initial phase for the first three months followed by 18-23 hours during the weaning phase for another three months. Then the FAB was worn for 12-18 hours a day during the maintenance phase until the patient was four years old .

Parents or guardians were asked to perform the stretching exercises for the patients, which included a passive range of motion of the involved ankle and foot at least 20 minutes twice a day. A squatting exercise was recommended in addition to the ankle stretching exercise after the walking age. The patients were followed at an outpatient clinic at a regular interval. Recurrence of CTEV was defined as a reappearance of at least one of the four components of CTEV $^{11,19}$.

Patient information extracted from medical record review included age at initiation of the treatment, gender, bilaterality, family geography, type of principal caregiver, and severity of CTEV at presentation. The severity of the deformity was graded from 1-4 according to the system of Dimeglio et $a l^{20}$. The patients were divided into two groups: grades 1-2 (mild to moderate) and grades 3-4 (severe to very severe). Treatment information included centre where the Ponseti method was initiated, compliance to FAB, compliance to the practice of stretching exercise, type of FAB, and complications of casting. Compliance to the use of $\mathrm{FAB}$ and stretching exercise were recorded in hours per day according to caregivers' self-report. Non-compliance to FAB was defined as the duration of FAB use of lesser than 23 hours, 18-23 hours, and 12-18 hours in the initial phase, weaning phase, and maintenance phase, respectively. Outcomes of interest were recurrence of CTEV, the duration between the initial correction and the detection of recurrence, and the need for recasting.

Data were reported as number and percent, or mean \pm standard deviation (SD) when appropriate. A case- control odds-ratio calculation was performed to evaluate parameters modelling for recurrence. Unadjusted odd ratios and $95 \%$ confidence interval $(\mathrm{CI})$ were calculated. P-value of less than 0.05 was considered statistically significant. A posthoc power analysis for a two-sample proportion was conducted. Statistical analysis was performed using Stata 13 (Stata Corp LP, College Station, TX, USA.). This study was approved by an ethical review board of the Faculty of Medicine, Chulalongkorn University, Thailand, COA 939/2017, and complied with the declaration of Helsinki 1975, as revised in 1983. For a retrospective study, formal inform consent was exempted.

\section{RESULTS}

The median age at initiation of the treatment was 3.4 (IQR; 2.1-12.6) with of 0.4 to 58.1 weeks. Six (18\%) patients had Dimeglio grade 2 (moderate) and $28(82 \%)$ patients had Dimeglio grades 3-4 (severe to very severe). The Ponseti method was initiated at our centre in $26(76 \%)$ patients. A median of six (range; 3-12) casts were applied and pTAL was performed in an outpatient setting in 32/34 (94\%) cases. All CTEV were clinically, fully corrected, defined as having a dorsiflexion at the ankle of $15^{\circ}$ and an abduction of the forefoot at $60^{\circ}-70^{\circ}$ after completion of the casting.

At an average follow-up period of $2.3 \pm 1.1$ (range; 0.6-4.2) years, $14 / 52(27 \%)$ feet in $11 / 34(32 \%)$ patients had recurrence at an average age of $26.4 \pm 11.9$ months. There were recurrent equinus in five feet and recurrent metatarsus adductus in nine feet. Non-compliance to the FAB protocol began at an average age of $11.2 \pm 6.5$ months. Odds ratio of patient- and treatment-associated factors related to the recurrence of CTEV are shown in Table I and II, respectively. Non-compliance to FAB during the weaning phase was significantly associated with recurrence with an odds ratio of 8.4 [95\% CI; (1.2-92.4), p=0.03]. Age of initiation of the treatment did not influence the recurrence. Non-parental caregivers, CTEV with Dimeglio grade 3-4, non-compliance to FAB during the initial and maintenance phases, lack of practice of stretching exercise and the use of fixed type bar also demonstrated higher but non-significant odds of higher chance of recurrence. No association was found between other clinical factors and the recurrence of CTEV. A post-hoc chi-square power analysis for a twosample proportion had a power of $87.3 \%$.

Ponseti casting was repeated in 11 patients (14 feet) with recurrent CTEV. Six feet responded to a repeat casting. A repeat pTAL was required in five feet with recurrent equinus, however, the plantigrade feet were achieved in $2 / 5$ feet. Limited posterior and plantar release procedures were performed on one foot. The caregivers of the remaining patient (two feet) refused further treatment due to their personal beliefs. Recurrent metatarsus adductus was flexible and correctable. The caregivers of the remaining patients 
Table I: Patients associated factors related to recurrence of idiopathic congenital talipes equinovarus post the Ponseti method

\begin{tabular}{|c|c|c|c|c|c|}
\hline Factors & & Recurrence & No Recurrence & OR (95\%Cl) & $\mathbf{P}$ \\
\hline Number of patients & & $11(32 \%)$ & $23(68 \%)$ & & \\
\hline Number of CTEV & & $14(27 \%)$ & $38(73 \%)$ & & \\
\hline Age at initiation of treatment & $\begin{array}{l}>12 \text { weeks } \\
<12 \text { weeks }\end{array}$ & $\begin{array}{l}3(33 \%) \\
8(32 \%)\end{array}$ & $\begin{array}{l}6(67 \%) \\
17(68 \%)\end{array}$ & $1.1(0.1-6.7)$ & 0.62 \\
\hline Gender & $\begin{array}{l}\text { Female } \\
\text { Male }\end{array}$ & $\begin{array}{l}4(33 \%) \\
7(32 \%)\end{array}$ & $\begin{array}{l}8(67 \%) \\
15(68 \%)\end{array}$ & $1.1(0.2-5.9)$ & 0.61 \\
\hline Bilaterality & $\begin{array}{l}\text { Bilateral } \\
\text { Unilateral }\end{array}$ & $\begin{array}{l}3(17 \%) \\
8(50 \%)\end{array}$ & $\begin{array}{l}15(83 \%) \\
8(50 \%)\end{array}$ & $0.2(0.03-1.19)$ & 0.07 \\
\hline Family geography & $\begin{array}{l}\text { Outside } \\
\text { Bangkok metropolitan area }\end{array}$ & $\begin{array}{l}7(41 \%) \\
4(23 \%)\end{array}$ & $\begin{array}{l}10(59 \%) \\
13(77 \%)\end{array}$ & $2.3(0.4-13.5)$ & 0.23 \\
\hline Type of principal caregiver & $\begin{array}{l}\text { Non-parents } \\
\text { Parents }\end{array}$ & $\begin{array}{l}2(40 \%) \\
9(31 \%)\end{array}$ & $\begin{array}{l}3(60 \%) \\
20(69 \%)\end{array}$ & $1.5(0.1-15.3)$ & 0.53 \\
\hline Severity at presentation & $\begin{array}{l}\text { Dimeglio 3-4 (feet) } \\
\text { Dimeglio } 2 \text { (feet) }\end{array}$ & $\begin{array}{l}13(30 \%) \\
1(11 \%)\end{array}$ & $\begin{array}{l}30(70 \%) \\
8(89 \%)\end{array}$ & $3.5(0.4-165.4)$ & 0.23 \\
\hline
\end{tabular}

Table II: Treatments associated factors related to recurrence of idiopathic congenital talipes equinovarus post the Ponseti method

\begin{tabular}{|c|c|c|c|c|c|}
\hline Factors & & Recurrence & No Recurrence & OR $(95 \% \mathrm{Cl})$ & $\mathbf{P}$ \\
\hline Number of patients & & $11(32 \%)$ & $23(68 \%)$ & & \\
\hline Number of CTEV & & $14(27 \%)$ & $38(73 \%)$ & & \\
\hline \multirow[t]{2}{*}{ Centre location } & Outside facility & $2(25 \%)$ & $6(75 \%)$ & $0.6(0.1-4.6)$ & 0.48 \\
\hline & $\mathrm{KCMH}$ & $9(35 \%)$ & $17(65 \%)$ & & \\
\hline \multirow[t]{2}{*}{ Compliance to $F A B$-initial phase } & No (<23 h/day) & $5(50 \%)$ & $5(50 \%)$ & $3.0(0.5-18.2)$ & 0.16 \\
\hline & Yes (23 h/day) & $6(25 \%)$ & $18(75 \%)$ & & \\
\hline \multirow[t]{2}{*}{ Wean phase } & No (<12 h/day) & $9(53 \%)$ & $8(47 \%)$ & $8.4(1.2-92.4)$ & 0.03 \\
\hline & Yes (12-18 h/day) & $2(11 \%)$ & $15(89 \%)$ & & \\
\hline \multirow[t]{2}{*}{ Maintenance phaset } & No (<12 h/day) & $11(40 \%)$ & $16(60 \%)$ & $10.5(0.5-201.8)$ & 0.08 \\
\hline & Yes (12-18 h/day) & 0 & $7(100 \%)$ & & \\
\hline Regular practice of & No & $3(75 \%)$ & $1(25 \%)$ & $8.3(0.5-452.1)$ & 0.09 \\
\hline stretching exercise & Yes & $8(27 \%)$ & $22(73 \%)$ & & \\
\hline \multirow[t]{2}{*}{ Type of FAB } & Fix (DB bar) & $8(42 \%)$ & $11(58 \%)$ & $2.9(0.5-20.8)$ & 0.27 \\
\hline & Articulated joint (Dobbs bar) & $3(20 \%)$ & $12(80 \%)$ & & \\
\hline \multirow[t]{2}{*}{ Complications of casting } & Yes (feet) & $5(42 \%)$ & $7(58 \%)$ & $2.5(0.5-11.6)$ & 0.19 \\
\hline & No (feet) & $9(23 \%)$ & $31(77 \%)$ & & \\
\hline \multirow[t]{2}{*}{ Need for recasting } & Yes (feet) & $3(33 \%)$ & $8(77 \%)$ & $1.0(0.1-5.3)$ & 0.62 \\
\hline & No (feet) & $11(25 \%)$ & $30(75 \%)$ & & \\
\hline
\end{tabular}

Data were presented as number of patient or feet (percent) as appropriate.

$\mathrm{Cl}$; confidence interval, CTEV; congenital talipes equinovarus, DB; Denis Browne, FAB; foot abduction brace, h; hours, KCMH; King Chulalongkorn Memorial Hospital, OR; odds ratio

tCase-control odds ratio uses a 0.5 correction in the cell that contains a zero.

(three feet) preferred stretching exercises instead of casting. This group of patients was closely monitored for a fixed deformity or a possible dynamic supination of the forefoot during the gait. An additional procedure, e.g. tibialis anterior tendon transfer, was not required during the follow-up period.

\section{DISCUSSION}

An excellent outcome of CTEV managed by the Ponseti method is well established. An initial correction has been achieved in the majority of the cases ${ }^{1,12,16}$ and it is confirmed by our experience in this study. However, recurrence of the deformity after correction does occur with a recurrent rate between $14-41 \%$ being reported ${ }^{6,9}$. In this retrospective review, non-compliance to the use of FAB to maintain $\mathrm{CTEV}$ correction was associated with a significant odds ratio of recurrence.

Bracing is an essential part of the Ponseti method. Standard protocol has been well outlined; however, many investigators note that bracing is a challenging process of the Ponseti method ${ }^{3}$. Previous studies have reported noncompliance rates to be $32-61 \%{ }^{9,19}$. Our study also had similar non-compliance rates. Dobbs et al used the criteria of noncompliance as complete discontinuation of the use of 
orthosis, which occurred during the first three months of bracing.

The parents or guardians often cited inconvenience as the main cause for not using the brace ${ }^{1,21}$. Moreover, the FAB should be worn until at least four years of age and early discontinuation of FAB was another known factor for recurrence ${ }^{22}$. Non-compliance to the FAB protocol occurred at approximately one year after initiating treatment. In our experience, this coincided with the period when the parents had to go back to work after the parental-leave period and had to transfer the care of the patient to other people including grandparents and day care personnel. To improve the parents' or guardians' awareness, standardised information explaining the Ponseti method and the importance of compliance to FAB should be emphasised to the parents or guardians at the start of treatment ${ }^{9,19}$. Regular discussion and support during each clinic visit and a followup phone call are very useful ${ }^{9,12}$.

Other factors that demonstrated higher but not significant odds ratio of recurrence include CTEV with Dimeglio grades 3-4 at presentation, not performing the stretching exercise, the use of fix bar and the principal caregiver of the patient was non-parental, e.g. grandparents, family-helpers or day care personnel. Age at initiation of the treatment did not associate with the recurrence of the deformity. This finding is consistent with the recent report by Awang et $a l^{23}$. Previous studies used the Dimeglio classification to quantify the severity of the deformities ${ }^{11,20,24}$. The Dimeglio grade 3 (severe) and grade 4 (very severe) may be associated with a higher chance of recurrence ${ }^{11,25}$. Dobbs et al reported that CTEV with Dimeglio grade 4 had an 8-fold chance of recurrence but this was not statistically significant ${ }^{1}$. Furthermore, Panjavi et al recently confirmed the association between severity of CTEV as rated by the Dimeglio classification and risk of recurrence ${ }^{11}$. This should remind the physician when applying the Ponseti method to this particular group of patients. On the other hand, Zhao et al devised a ratio of correction improvement using the Pirani scoring system and linked that to the risk of recurrence ${ }^{6}$.

Kite cited common errors of treatment that physicians relied on "Brace Maker" and "family exercise"13. The Ponseti method recommended stretching exercise performed by the parents or guardians as part of the daily care ${ }^{1,11}$. We currently focus on routine stretching and squatting exercises to be performed when the patient has reached the walking age. We anticipate that this strategy may lessen the chance of recurrence as shown in previous reports ${ }^{2,11}$. A recent study demonstrated that routine stretching exercise of the foot and ankle could be an important part of maintaining range of ankle and subtalar motions after a soft tissue release procedure for $\mathrm{CTEV}^{17}$. Another factor that can affect the recurrence is the type of FAB used ${ }^{18}$. Non-compliance to the $\mathrm{FAB}$ will definitely increase the recurrence rate of CTEV. We observed comparable odds of recurrence in the group of patients with fixed type bar and articulated type bar. The Denis Browne bar was preferred during the Ponseti era ${ }^{4}$ and it is still the primary choice of FAB in many centres ${ }^{11,26}$. Our findings could be explained by the recent study by Agarwal et al who reported dynamic change of foot abduction measurements and successful outcomes utilising fixed FABSteenbeek type ${ }^{7}$. On the other hand, Chen et al reported a higher parental compliance rate when the articulated bar was first introduced ${ }^{27}$ and subsequently confirmed by Garg et al in a dynamic FAB model ${ }^{18}$. Application of foot pieces and the bar connector to a patient is easier when using the articulated type bar. The articulated type bar allows the knees and hips movements and could lower the blister formation $^{18,27}$. The major obstacle in our setting is the cost of commercial articulated bar as experienced by others ${ }^{2,3,9,21}$. Before the treatment, the advantages and disadvantages of each type of FAB should be discussed with the parents or guardians. Janicki et al emphasised that the FAB should not be replaced by an ankle foot orthosis. The use of ankle foot orthoses demonstrated a notable recurrence rate when compared to the use of $\mathrm{FAB}^{26}$.

Family or domestic helper is common in our society, and corroborated by other studies conducted in different cultural background $^{10,28}$, and could dominate parental decision ${ }^{2}$. In contrast, Ramirez et al did not find any correlations between the parents' or guardians' factors and compliance rate ${ }^{15}$. According to our experience, it may be helpful to include other family members (e.g. grandparents) into the treatment scheme. This supports a recent work by Malagelada et al that bracing period could affect the entire family and recommended a supporting system to lower the burden ${ }^{28}$.

Despite the best effort, recurrences of CTEV do occur. Encouraging the parents or guardians and caregivers to adhere to the FAB protocol could lower the chance of recurrences ${ }^{29}$. A daily physiotherapy program adjunct to the Ponseti method shows an improvement of the Dimeglio scores $^{30}$. Recurrent metatarsus adductus is prevalent in our study, corroborated by results from other studies ${ }^{31,32}$. Expectant treatment could be employed in flexible deformities. A repeat episode of Ponseti casting was successfully utilised ${ }^{4,33}$. Transfer of tibialis anterior tendon to lateral column of the foot improves long-term functions when dynamic forefoot supination observed during gait is evident $^{34}$. The range of ankle dorsiflexion is related to the outcomes thus recurrent ankle equinus needs more aggressive management ${ }^{35}$. If recurrent heel varus combined with ankle dorsiflexion of less than $15^{\circ}$ is detected, we prefer repeat casting and pTAL as advocated by Dobbs ${ }^{33}$. Marquez et al recommended a repeat Ponseti casting followed by a short leg walking cast until the range of ankle dorsiflexion of $20^{\circ}$ was attained ${ }^{35}$. A soft tissue release procedure may be inevitable. It should follow an "a la carte" concept and progressively correct the remaining deformities and avoid 
dissection into the subtalar complex as possible ${ }^{17,33}$. Several types of osteotomy around the midfoot have been proposed if the deformities become fixed ${ }^{32}$.

A few limitations need to be addressed. First, the mean 2.3 years of follow-up of the study is shorter than the recommended 4-year duration of $\mathrm{FAB}^{1,4,8}$. Moreover, due to the nature of the retrospective study, compliance to the treatment relied on the parents' or guardians' report. The prevalence of non-compliance to FAB could be higher in a longer-term follow-up. More recurrent CTEV could develop approaching the end of the FAB period and additional surgical procedures are a possibility. Events occurred in some parameters studied were not significant which may be due to the small sample size. Recruiting more CTEV patients would give an insight appraisal of other risk factors.

\section{CONCLUSION}

In conclusion, non-compliance to FAB is related to the risk of CTEV recurrence after the Ponseti method. Family education and support are pivotal and physicians' awareness is equally important. The parents and guardians should be encouraged to comply with the Ponseti method to decrease the risk of recurrence.

\section{CONFLICT OF INTERESTS}

The authors declare that there is no conflict of interest.

\section{ACKNOWLEDGEMENT}

We thank Ms. Pirapon June Ohata for her assistance in editing and proofreading the manuscript.

\section{REFERENCES}

1. Dobbs MB, Rudzki JR, Purcell DB, Walton T, Porter KR, Gurnett CA. Factors predictive of outcome after use of the Ponseti method for the treatment of idiopathic clubfeet. J Bone Joint Surg Am. 2004; 86(1): 22-7.

2. Evans AM, Chowdhury MM, Kabir MH, Rahman MF. Walk for life - the national clubfoot project of Bangladesh: The four-year outcomes of 150 congenital clubfoot cases following Ponseti method. J Foot Ankle Res. 2016; 9: 42.

3. Morcuende JA, Cook TM. The Ponseti method in low and middle income countries: Challenges and lessons learned. Foot Ankle Clin. 2015; 20(4): 547-54.

4. Ponseti IV, Smoley EN. The classic: Congenital club foot: The results of treatment. 1963. Clin Orthop Relat Res. 2009; 467(5): 1133-45.

5. Rasit A, Rasit A, Azani H, Zabidah P, Merikan A, Nur Alyana B. Clubfoot: The treatment outcome using quantitative assessment of deformity. Malays Orthop J. 2012; 6(SupplA): 2-5.

6. Zhao D, Li H, Zhao L, Kuo KN, Yang X, Wu Z, et al. Prognosticating factors of relapse in clubfoot management by Ponseti method. J Pediatr Orthop. 2018; 38(10): 514-20.

7. Agarwal A, Kumar A, Mishra M. The foot abduction characteristics following Steenbeek foot abduction brace. J Orthop Surg (Hong Kong). 2017; 25(1): 1-7.

8. Ponseti International. Bracing tips. 2018 [cite March 14, 2018]. Available from: http://www.ponseti.info/bracing-tips.html.

9. Zionts LE, Dietz FR. Bracing following correction of idiopathic clubfoot using the Ponseti method. J Am Acad Orthop Surg. 2010; 18(8): 486-93. doi: 10.5435/00124635-201008000-00005

10. Jawadi AH, Al-Abbasi EM, Tamim HA. Factors predicting brace noncompliance among idiopathic clubfoot patients treated with the Ponseti method. J Taibah Univ Med Sci. 2015; 10(4): 444-8.

11. Panjavi B, Sharafatvaziri A, Zargarbashi RH, Mehrpour S. Use of the Ponseti method in the Iranian population. J Pediatr Orthop. 2012; 32(3): e11-4.

12. Haft GF, Walker CG, Crawford HA. Early clubfoot recurrence after use of the Ponseti method in a New Zealand population. $J$ Bone Joint Surg Am. 2007; 89(3): 487-93.

13. Kite JH. Conservative treatment of the resistant recurrent clubfoot. Clin Orthop Relat Res. 1970; 70: 93-110. doi: 10.1097/00003086-197005000-00011

14. Moon DK, Gurnett CA, Aferol H, Siegel MJ, Commean PK, Dobbs MB. Soft-tissue abnormalities associated with treatmentresistant and treatment-responsive clubfoot: Findings of MRI analysis. J Bone Joint Surg Am. 2014; 96(15): 1249-56. 
15. Ramirez N, Flynn JM, Fernandez S, Seda W, Macchiavelli RE. Orthosis noncompliance after the Ponseti method for the treatment of idiopathic clubfeet: A relevant problem that needs reevaluation. J Pediatr Orthop. 2011; 31(6): 710-5.

16. Avilucea FR, Szalay EA, Bosch PP, Sweet KR, Schwend RM. Effect of cultural factors on outcome of Ponseti treatment of clubfeet in rural America. J Bone Joint Surg Am. 2009; 91(3): 530-40.

17. Limpaphayom N, Kerr SJ, Prasongchin P. Idiopathic clubfoot: Ten year follow-up after a soft tissue release procedure. Int Orthop. 2015; 39(1): 81-6.

18. Garg S, Porter K. Improved bracing compliance in children with clubfeet using a dynamic orthosis. J Child Orthop. 2009; 3(4): 271-6.

19. Zhao D, Liu J, Zhao L, Wu Z. Relapse of clubfoot after treatment with the Ponseti method and the function of the foot abduction orthosis. Clin Orthop Surg. 2014; 6(3): 245-52.

20. Dimeglio A, Bensahel H, Souchet P, Mazeau P, Bonnet F. Classification of clubfoot. J Pediatr Orthop B. 1995; 4(2): 129-36.

21. Yoyos DI. A four-year review of delayed initial treatment of patients with congenital talipes equinovarus in a general hospital. Malays Orthop J. 2015; 9(1): 11-3.

22. Sangiorgio SN, Ebramzadeh E, Morgan RD, Zionts LE. The timing and relevance of relapsed deformity in patients with idiopathic clubfoot. J Am Acad Orthop Surg. 2017; 25(7): 536-45.

23. Awang M, Sulaiman AR, Munajat I, Fazliq ME. Influence of age, weight, and Pirani score on the number of castings in the early phase of clubfoot treatment using Ponseti method. Malays J Med Sci. 2014; 21(2): 40-3.

24. Flynn JM, Donohoe M, Mackenzie WG. An independent assessment of two clubfoot-classification systems. J Pediatr Orthop. 1998; 18(3): 323-7.

25. Azarpira MR, Emami MJ, Vosoughi AR, Rahbari K. Factors associated with recurrence of clubfoot treated by the Ponseti method. World J Clin Cases. 2016; 4(10): 318-22.

26. Janicki JA, Wright JG, Weir S, Narayanan UG. A comparison of ankle foot orthoses with foot abduction orthoses to prevent recurrence following correction of idiopathic clubfoot by the Ponseti method. J Bone Joint Surg Br. 2011; 93(5): 700-4.

27. Chen RC, Gordon JE, Luhmann SJ, Schoenecker PL, Dobbs MB. A new dynamic foot abduction orthosis for clubfoot treatment. J Pediatr Orthop. 2007; 27(5): 522-8.

28. Malagelada F, Mayet S, Firth G, Ramachandran M. The impact of the Ponseti treatment method on parents and caregivers of children with clubfoot: A comparison of two urban populations in Europe and Africa. J Child Orthop. 2016; 10(2): 101-7.

29. Miller NH, Carry PM, Mark BJ, Engelman GH, Georgopoulos G, Graham S, et al. Does strict adherence to the Ponseti method improve isolated clubfoot treatment outcomes? A two-institution review. Clin Orthop Relat Res. 2016; 474(1): 237-43.

30. Nilgun B, Suat E, Engin SI, Fatma U, Yakut Y. Short-term results of intensive physiotherapy in clubfoot deformity treated with the Ponseti method. Pediatr Int. 2011; 53(3): 381-5.

31. Bhaskar A, Patni P. Classification of relapse pattern in clubfoot treated with Ponseti technique. Indian J Orthop. 2013; 47(4): 370-6.

32. Parsa A, Moghadam MH, Jamshidi MH. Relapsing and residual clubfoot deformities after the application of the Ponseti method: A contemporary review. Arch Bone Jt Surg. 2014; 2(1): 7-10.

33. Dobbs MB, Gurnett CA. Update on clubfoot: Etiology and treatment. Clin Orthop Relat Res. 2009; 467(5): $1146-53$.

34. Holt JB, Oji DE, Yack HJ, Morcuende JA. Long-term results of tibialis anterior tendon transfer for relapsed idiopathic clubfoot treated with the Ponseti method: A follow-up of thirty-seven to fifty-five years. J Bone Joint Surg Am. 2015; 97(1): 47-55.

35. Marquez E, Pacey V, Chivers A, Gibbons P, Gray K. The Ponseti technique and improved ankle dorsiflexion in children with relapsed clubfoot: A retrospective data analysis. J Pediatr Orthop B. 2017; 26(2): 116-21. 